\title{
A Pilot Recycling of Plastic Pure Water Sachets/Bottles into Composite Floor Tiles: A Case Study from Selected Dumping Site in Ogbomoso
}

\section{Temitope $\mathrm{AK}^{1 *}$, Abayomi $\mathrm{OO}^{2}$, Ruth $\mathrm{AO}^{3}$ and Adeola $\mathrm{AP}^{4}$}

${ }^{1}$ Department of Mechanical Engineering, Ladoke Akintola University of Technology, Ogbomoso, Nigeria

${ }^{2,4}$ Department of Industrial and Production Engineering, University of Ibadan, Oyo State, Nigeria

${ }^{3}$ Department of Chemical Engineering, Ladoke Akintola University of Technology, Ogbomoso, Nigeria

\begin{abstract}
The adverse effect associated with the surge in uses of non-biodegradable plastic products include the blockage of drains, suffocating some animal life, who accidentally take them as food, grounds impermeable to water and several other hazards. The need then arises to finding a safe means converting these plastic waste products into other useful and harmless items.

The method adopted involves the collection of the plastic materials from trenches, drainages, streets, dump sites and from eateries around Ogbomoso metropolis. These were sorted and washed before the Shredding Process was carried. Melting of the Shredded Plastics and poured into a dimensioned wooden mould and Cured for some days.

It could be shown from the crushing test that the conventional tiles fail under slight heavier loads of $34 \mathrm{kN}$ as comparable to the $29 \mathrm{kN}$ failure load of the composite tiles. However the water absorption test has no effect on both the conventional and the composite tiles respectively. Furthermore, the flammability test carried out on the conventional tiles shows a slight change as compared to the composite tiles which split under the effect of flame. Moreover the composite tiles recorded a higher value than that of the conventional tiles. The tests show that the composite floor tiles are cheaper, durable, and affordable as compared to the ceramic tile. Hence, the performances of the composite floor tiles though are a little less than that of the commercial ceramic floor tiles yet appear a reasonably good substitute at more reasonable rate.
\end{abstract}

Keywords: Composite floor tiles; Conventional tiles; Plastic waste; Recycling; Sawdust; Flammability

\section{Introduction}

Plastic is defined as synthetic or semi-synthetic materials which are polymeric and are composed of large molecules of organic substances known as monomers. The large molecules that are formed during a process known as polymerization are known as polymers [1].

The major divisions of plastics are thermoplastic and the thermosetting polymers. Thermoplastics are the form of plastics that do not undergo chemical changes in their composition when subjected to heat and can be remoulded into another shape even after solidification. Thermosetting on the other hand are non-recycled polymers which undergo an irreversible chemical changes when subjected to heat, they melts and take a shape once after which they cannot be moulded into another shape. The role of plastics in human lives cannot be over emphasized ranging from use as household appliances, packaging materials, potable water and beverage containers, kitchen utensils, furniture, toys, automobile parts, polythene bags etc [2]. Attributed the great attention given to their use as due to their generally light, cheap and durability nature and this account for their preference over other materials [3].

Pure water sachets are products of plastics which are used widely in Nigeria for various purposes, among such function is in the packaging of pure potable water. This when consumed and disposed can pose a great environmental problem if not disposed properly. Empty polythene plastic containers are rampant in the nook and cranny of the Nigerian landscape. Plastic materials are non-biodegradable material and when find their way into the soil prevents water from getting to the root of the plants and also restrict the stretching of the plant root. Some of it hinders the exchange of gases for respiration by plants and animals in the water body. The entire length of road from Lagos State to Benin lined with millions of old and used containers deposited by travellers. Some of these wastes that litters the streets find their ways into drains, trenches and canals, thereby clogging drains and hindering the free flow of water and consequently leading to flooding [4]. The expanding population and increased preference for packaged commodities are worsening the negative environmental impacts of these plastics [4].

In view of all the hazards that accompany the improper disposal of plastic wastes, the need then arise for an alternative means to manage the plastics waste. The only means of adequately take care of several tonnes of plastic wastes being disposed is through the adoption of recycling process. This recycling process will go a long way to contribute to a cleaner environment. Plastic recycling is the process of recovering plastic wastes and turning old or scrap plastic into useable products that can re-enter the manufacturing chains. This will in turn generate revenue, create more job opportunities and reduce the hazards associated with improper disposal of plastic wastes [3].

*Corresponding author: Temitope AK, Department of Mechanical Engineering Ladoke Akintola University of Technology, Ogbomoso, Nigeria, E-mail: academicpapers4real@gmail.com

Received August 13, 2015; Accepted August 22, 2015; Published August 30, 2015

Citation: Temitope AK, Abayomi OO, Ruth AO, Adeola AP (2015) A Pilot Recycling of Plastic Pure Water Sachets/Bottles into Composite Floor Tiles: A Case Study from Selected Dumping Site in Ogbomoso. J Material Sci Eng 4: 201. doi:10.4172/2169-0022.1000201

Copyright: (c) 2015 Temitope AK, et al. This is an open-access article distributed under the terms of the Creative Commons Attribution License, which permits unrestricted use, distribution, and reproduction in any medium, provided the original author and source are credited. 


\section{Literature Review}

\section{Plastic}

Plastic is a type of synthetic or non-synthetic polymer, similar in many ways to natural resins found in trees and other plants. Polymers can be defined as any of various complex organic compounds produced by polymerization, capable of being moulded, extruded, cast into various shapes and films or drawn into filaments and can then be used for manufacturing textile fibres [5].

According to Fagbemiro, describe plastics as polymers. Polymers can be defined as materials made from many units [6]. Polymer is seen as a chain which is made up of various smaller units. These units could be carbon, hydrogen, oxygen and silicon.

\section{Recycling of plastics wastes}

Plastic recycling is the process of recovering scrap or waste plastics and refining the material into useful products, sometimes completely different in form from their original state [7]. In years past, various works have been done on recycling of plastic wastes by many researchers. According to Kalilu, attributed that large quantities of horticultural, domestic and industrial wastes are generated and deposited in landfills [4]. The horticultural wastes are biodegradable while a good number of the industrial wastes are not and as thus, will pose threat to health, drainage and urban planning.

Owing to the rapid upsurge in the price of building materials, a young man from Kodungallur, in Thrissur district in India devised a way of making floor tiles from plastic waste. First, the plastic waste is being powdered in a machine after which it is mixed with liquid asphalt and chipped metal before being heated. This type of tiles is seen to have numerous advantages over conventional cement tiles. Some of the advantages are that the plastic tiles are relatively cheap, requires less time for production and relatively lighter when compared to the cement tiles [8].

Advantages of recycling plastic wastes: Recycling of plastic wastes has so many advantages to human being and the ecosystem when compared with all other methods of solid waste management adopted. Some of the numerous advantages of recycling of plastic wastes are:

- Reduction of the use of fresh raw materials [7].

- Conservation of energy used for production of fresh products.

- It creates job opportunities [9].

- Emission of poisonous gases such as carbon-dioxide, carbonmonoxide, nitrogen-oxide and sulphur-oxide are reduced [10].

- Conservation of non-renewable fossil fuels- plastic production uses $8 \%$ of the world's oil production, $4 \%$ as feedstock and $4 \%$ during manufacture [10].

- As plastic wastes that litter the streets and choke drainages which serve as breeding ground for mosquitoes and flies are reduced, diseases associated with these vectors will also reduced [2].

Plastic wastes recycling techniques: There are basically two types of plastic wastes recycling techniques. They are:

- Mechanical recycling techniques and

- Chemical recycling techniques.
Mechanical recycling techniques: It comprises of some processes, which are shredding, melting and pelletizing of waste plastics. Plastics must be sorted prior to mechanical recycling techniques. Mostly, sorting is done manually. Advancements have been made in sorting plastics. The technology introduced in sorting plastics automatically includes using X-ray fluorescence, infrared and near infrared spectroscopy, electrostatics and flotation. These sorting techniques are used in large scale recycling. Owing to the expensive nature of this sorting technology, sorting will be done manually. After the sorting exercise, the plastics are shredded into smaller sizes (between $5 \mathrm{~mm}^{2}-$ $10 \mathrm{~mm}^{2}$ ). The shredded plastics are melted and moulded directly into a new product or melted and formed into pellets or granules [10].

Chemical or feedstock recycling techniques: Feedstock recycling technique describes a range of plastic recovery procedures to make plastics, which breaks down polymers into their constituent monomers, which in turn can be used again in refineries, or petrochemical and chemical production. Some of the numerous ranges of feedstock recycling techniques currently being explored include: (i) Pyrolysis, (ii) Hydrogenation, (iii) Gasification and (iv)Thermal cracking.

Feedstock recycling has a greater flexibility over composition and is more tolerant to impurities than mechanical recycling. It is very expensive and requires very large quantities of used plastic for reprocessing to be economically viable [10].

\section{Wood}

Wood is the hard fibrous substance composing mostly of the stem and branches of a tree or shrub and covered by the bark. The inner core of the wood is called the heartwood while outer layer is called the sapwood [11].

Uses of wood: Wood is one of the most useful and versatile materials available. It has found suitability for general use due to the advantages it has over other materials.

Some of the various uses of wood are listed below:

- It is used as fuel: Wood has valuable quantities as a fuel and is clean, efficient and renewable energy source. Wood fuel may be available as firewood, charcoal, chips, sheets, pellets, and sawdust.

Basically, when burnt, they provide a great amount of heat energy. This energy can be used for cooking and heating, and occasionally for fuelling steam engines and steam turbines that generate electricity.

- Construction: Wood is an important construction material since human began building shelters, bridges and boats. It has found use in the construction of scaffolds, mould, frames, interior doors, roof construction and exterior cladding.

Sawdust as waste product: Generally, wood is biodegradable [12]. Before a desired finished product is derived from wood, it has to go through so many processes. Some of these conversion processes include falling down of the tree, debarking, sawing, sanding, milling, lathing, drilling, chipping and carving. During the conversion process, off cuts are generated which pose as nuisance and are seen as having limited use to human being. These off cuts produced tend to attract pests and micro-organisms to wherever they are found. They cause infections and sicknesses to human beings if found around the home.

\section{Methodology}

Materials and equipment used in the production of composite tiles: The materials required for this research work were 
sourced locally. The composite floor tile was produced from through the blending of water sachets, bottles and saw dusts. Other materials include charcoal, cardboards.

The equipment used in this work is listed below:

- eighing Scale

- Hydraulic Hot Press

- Metal Slab

- Measuring Cylinder

- Hand Trowel

- Sieve

- Hand glove

- Wood Stirrer

- Coal pot

- Mouth mask

- Scissors

- Razors

- Knives

\section{Procedure into pilot scale production of composite floor tiles} below:

The steps involved in the production of the floor tiles are detailed

Collection of raw materials: The plastic materials were collected from trenches, drainages, streets, dump sites and from eateries around Ogbomoso metropolis. The reason for using this area as case study is due to large volume of plastics wastes generated by these selected area. To effectively collect these plastic wastes the areas where plastics wastes are generated in large quantities were identified.

Sorting, washing and shredding process: This involves the separation of plastic materials into categories and this is done according to the Plastic Identification Code (PIC). This is done in bigger establishment with the aid of an automated sorting system. However for this work a manual sorting system was employed for the pilot scale production.

Detergent and water was used to wash the already sorted plastic materials and thereafter leave to dry. The reason for washing process is to remove label attached to bottles and to get rid of contaminants such as adhesives and dirt. However, the shredding process in a small scale recycling process employs the rapid granular method in shredding the dried sorted plastic materials. It is however worthy of note that the shredding process was carried out with the aid of scissors.

Collection of a sun-dried sawdust and sieving operation: The waste products from saw dusts were collected during wood milling operations of oganho, gmelina. Mahogany and iroko tree. After the various types of sawdust were collected, they were placed separately, directly under the sun. This was to dehydrate the sawdust for just one day, being in powdery form, the drying was quite fast. The sun-dried saw dust was sieved to different degree of fineness. The fine particles were used to provide the fine surface finishing while the larger particles were used to give a rough/coarse surface finish.

Melting of the shredded plastics and curing process: An aluminium pot is placed on a heat source (charcoal) for close to 3 minutes and $320 \mathrm{~g}$ of the shredded water sachet and other plastic waste component inclusive. The shredded material is then allowed to melt completely inside the heated aluminium pan. A $100 \mathrm{~g}$ of the finely defined sun-dried saw dust is then added to the mixture. The mixture is stirred to allow for uniformity of the blended mixture. This is then poured into a pre-designed wooden mould that has a card board for the interior. The edge of the mould were gently banged on the ground continuously, this is to allow for even spreading of the molten material which aids the escape of bubbles that can cause a crack in the composite. The curing process involves the cooling of the composite material to allow for solidification. This is done for 20 minutes in air (Figure 1).

\section{Laboratory tests on the composite produced}

Tests were carried out on the composite samples to ascertain their suitability for floor tiling and compared with the mechanical tests on the conventional tiles. These tests include the water absorption test, crushing test, frictional test, and the flammability test.

Water absorption (Cold and hot testing): In cold absorption testing, the weight of the composite and conventional tile before immersion into the cold water was determined using a scale of capacity of $5 \mathrm{~kg}$. After 24 hours of immersion, the weight of both the pilot produced composite and conventional tiles was taken again.

However the hot absorption test involves weighting the composite and conventional sample using the weighing scale and thereafter dipping into boiling water for 30 minutes. A stone was then placed on the composite and conventional material to prevent it from floating. After 30 minutes the composite was reweighed and the quantity of water absorbed was obtained.

Crushing test: The Universal Testing Machine (U.T.M) was used to carry out this test. Here the dimension of the composite and conventional material were reduced to $100 \mathrm{~mm}$ in length and breadth and thickness is of $35 \mathrm{~mm}$. This was placed in the machine and loading was applied until there is a noticeable fracture in the composite and conventional material. The failure load was thereby obtained.

Frictional coefficient test: The frictional coefficient test is used to obtain the slip properties of the tiles produced, this is done by placing the composite and conventional sample on a level platform, and a known mass of match box, toothpaste tube and a stone was placed on the composite. With one end of the material in position, the opposite end of the composite was continuously raised such that the material inclines at an angle. The angle of inclination was continuously increased until the mass placed on top of the composite rolled off. At this point the value of the angle of inclination was taken; this is called

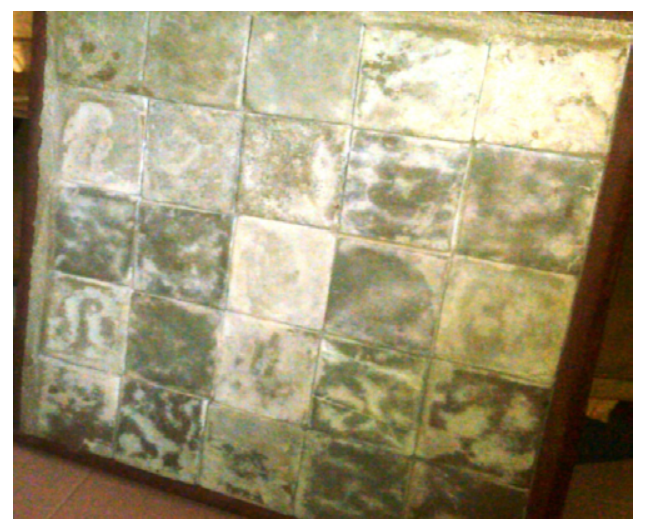

Figure 1: A Panel of the composite tiles produced from the recycled plastic materials. 
the angle of repose. This was done using different materials of different weight and surface finish.

The Frictional coefficient is obtained using the equation below:

Frictional coefficient $\mu(\mathrm{N} / \mathrm{Kg})=\operatorname{Tan} \theta$

\section{Flammability tests (Composite and conventional)}

- Composite testing: The composite sample was place in the direction of heat from an oxy-acetylene gas for about one second. The process was repeated but the duration of the release of heat on the composite was increased to 5 seconds.

- Conventional testing: A piece of conventional tile was thrown into the fire and left for 2 minutes before taken out of the fire. This was repeated for duration of 2 minutes and observation was made for both cases.

\section{Results and Discussion}

\section{Results from water absorption cold and hot testing}

Cold testing: The following results were obtained for the cold absorption test carried out:

Weight of the composite before emersion $\mathrm{A}=0.32 \mathrm{~kg}$

Weight of the composite sample after cold immersion $B=0.32 \mathrm{~kg}$

Amount of water absorbed $=$ Weight after immersion - weight before emersion $=\mathrm{B}-\mathrm{A}=0.32-0.32=0.00 \mathrm{~kg}$

From the above result the composite tiles produced from the recycled plastic has a zero absorbing capacity when immersed in cold water. This is comparable to the conventional ceramic tiles sold in the market, which also had a water resistant. Hence, the composite tiles produced from the recycled plastic bottles and water sachet can act as an alternative in place of the ceramic tiles.

Hot testing: The amount of water absorbed during the hot absorption test was obtained as follow at:

Weight of the composite before emersion $\mathrm{A}=0.32 \mathrm{~kg}$

Weight of the composite sample after hot immersion $B=0.32 \mathrm{~kg}$

Amount of water absorbed $=$ Weight after immersion - weight before emersion $=\mathrm{B}-\mathrm{A}=0.32-0.32=0.00 \mathrm{~kg}$

From the calculation above, it can be observed that there was no water absorption by the composite after 30 minutes of immersion in boiling water. Also there was no crack on the composite sample tested, this show the ability of the tiles to resist heat from the boiling water.

With the above observation, it is pertinent to note that, the composite produced from a recycled water sachet/plastic bottles possess the ability to resist heat from the boiling water. Hence the composite tiles can be used as floor and wall tiles for both bathroom and kitchen.

\section{Results from the crushing test}

The value of the failure load of the composite was $34 \mathrm{KN}$ which is considerable and this is a reason why the composite can be seen as an alternative for tile production. For a material to be used as floor tile it must have high load carrying capacity as people and items of various weight will be stationary or moving on the tile. Also, it was also noticed that the composite did not shatter under sudden loading. Hence, it has high ductility. This is one major setback for the conventional tiles which will readily shatter into new smaller sharp pieces under sudden loading or when released from a height and these can cause serious injuries to human beings.

\section{Results from frictional coefficient test}

The different material used for the frictional coefficient tests were toothpaste tube, match box, and stone. Their weight and coefficient of friction are calculated as shown in Tables 1 and 2. Given that the angle of repose as $\emptyset$, weight of material as $L$, and the coefficient of friction as $\mu$ (Figure 2).

Comparing the values of frictional coefficients of the composite and that of the conventional tiles, it can be deduced that the composite has higher frictional coefficient than the conventional tiles and this will promote more friction. For this reason the composite tiles produced from the recycled plastics can be used as home floor tiles and reduced the risk of slipping on tiles which could cause injury to human being.

\section{Results from flammability tests (Composite and conventional)}

- Test on composite tiles: It was observed that after heat was supplied to the surface of the composite for durations of 1 second and 5 second separately, and the heat was taken away, there was just a little noticeable change in the surface of the composite. This means that the composite did not burn after the heat was taken away from. It can therefore be concluded that

\begin{tabular}{|l|l|l|l|}
\hline Material & $\begin{array}{l}\text { Angle of repose } \\
(\boldsymbol{\varnothing})\end{array}$ & $\begin{array}{l}\text { Weight of } \\
\text { Material ( L) }\end{array}$ & $\begin{array}{l}\text { Frictional coefficient } \\
\boldsymbol{\mu}(\mathbf{N} / \mathbf{k g})\end{array}$ \\
\hline Toothpaste tube & 17.5 & 0.040 & 0.315 \\
\hline Match box & 24 & 0.020 & 0.445 \\
\hline Stone & 26 & 0.080 & 0.488 \\
\hline
\end{tabular}

Table 1: Determine the Frictional Coefficient for different materials on the Composite tile.

\begin{tabular}{|l|l|l|l|}
\hline Material & Angle of repose $ø$ & $\begin{array}{l}\text { Weight of Material } \\
\text { (L) }\end{array}$ & $\begin{array}{l}\text { Frictional } \\
\text { coefficient } \boldsymbol{\mu ( N / k g )}\end{array}$ \\
\hline Toothpaste tube & 16.5 & 0.040 & 0.296 \\
\hline Match box & 15 & 0.020 & 0.268 \\
\hline Stone & 19 & 0.080 & 0.344 \\
\hline
\end{tabular}

Table 2: Determine the Frictional Coefficient for different materials on the Conventional tile.

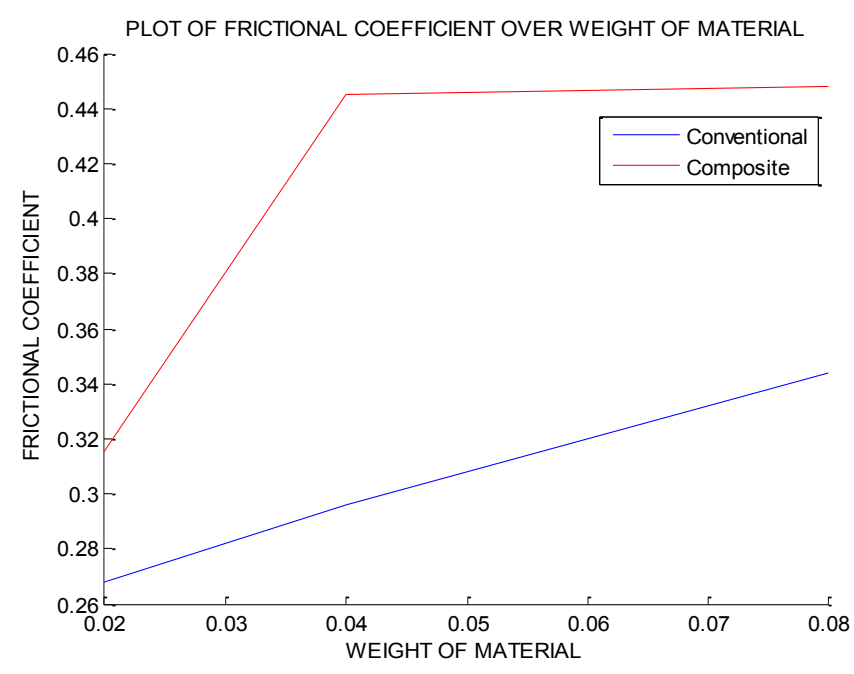

Figure 2: A graph of material weight against frictional coefficient. 
Citation: Temitope AK, Abayomi OO, Ruth AO, Adeola AP (2015) A Pilot Recycling of Plastic Pure Water Sachets/Bottles into Composite Floor Tiles: A Case Study from Selected Dumping Site in Ogbomoso. J Material Sci Eng 4: 201. doi:10.4172/2169-0022.1000201

Page 5 of 5

\begin{tabular}{|l|l|l|}
\hline Mechanical Tests & Conventional Tiles & Composite Tiles \\
\hline Crushing Test & $34.00 \mathrm{kN}$ & $29.00 \mathrm{kN}$ \\
\hline Water Absorption Cold Tests & $0.00 \mathrm{~kg}$ & $0.00 \mathrm{~kg}$ \\
\hline Water Absorption Hot Tests & $0.00 \mathrm{~kg}$ & $0.00 \mathrm{~kg}$ \\
\hline Flammability Tests & Slight changes flame & Split under flame \\
\hline Average Frictional Coefficient & $0.303 \mathrm{~N} / \mathrm{kg}$ & $0.416 \mathrm{~N} / \mathrm{kg}$ \\
\hline
\end{tabular}

Table 3: Summary of Some of the Mechanical Test Carried Out.

the composite has less flammability. This is an advantage for the composite as it makes it good substitute for floor tiling. This also means that on exposure of the composite to fire hazards like in the case of fire incidence in homes, it will take considerable time before the composite will burn off.

- Test on conventional tiles: It was observed that the conventional floor tile forcefully split into two separate pieces after it was placed in the fire at duration of 2 minutes. For composite tile, it started burning at duration of just 10 seconds but was still left in the fire for additional 1 minute 50 seconds. It was observed that only few part of the surface of the composite burnt and the composite did not split into 2 pieces forcefully as in the case of the conventional tile. This is an advantage for the composite tile as in a case of fire incidence. Also, the composite tile will have tendency of being reused as it will not spoil into separate pieces and all that is needed to be done is to refine the surface of the composite for a smooth surface. Furthermore, the composite tile will not cause injuries in the case of inferno as they will not forcefully split into pieces that will pierce into human skins.

It could be shown from Table 3 the crushing test that the conventional tiles fail under slight heavier loads of $34 \mathrm{kN}$ as comparable to the 29 $\mathrm{kN}$ failure load of the composite tiles. However the water absorption test has no effect on both the conventional and the composite tiles respectively. Furthermore, the flammability test carried out on the conventional tiles shows a slight change as compared to the composite tiles which split under the effect of flame. Moreover the composite tiles recorded a higher value than that of the conventional tiles.

\section{Conclusion}

It was concluded that plastic wastes (pure water sachets and plastic bottles) which pose hazards to human beings and the environment can be converted into useful plastic based products such as utensils, plastic chairs, key holders, infant bench, flower vase, tiles and buttons by adopting a simple recycling method which can be carried out by small cottage industries. It is recommended that recycling should be adopted as the main method plastic waste management as it has various advantages such as reduction of the hazards that improper waste management constitute and also to reduce the tapping into fresh raw materials for development of products over the methods of plastic waste management.

\section{References}

1. Hazzan EB (2003) A Study of Plastic Moulding Techniques in Oyo State. Ladoke Akintola University of Technology, Ogbomoso, Oyo State.

2. Abota CA (2012) Recycling of plastic wastes in Ghana; A way to reduce environmental problems/ pollutions. University of Ghana, Accra, Ghana.

3. Hopewell J, Dvorak R, Kosior E (2009) Plastics recycling: Challenges and opportunities. Phil Trans R Soc B 364: 2115-2126.

4. Kalilu RO (2013) Art from art for art: Conceptualising existence in the space of the visual Arts. Lautech Printing Press.

5. (2013) American Chemistry Council Life Cycle of a Plastic Product.

6. Fagbemiro SS (2001) Mathematical modelling of a batch for the production of fuel oil from waste polyethylene materials. Ladoke Akintola University of Technology, Ogbomoso, Oyo State.

7. (2001) An Introduction to Plastics

8. Naryanan KS (2007) Making Floor Tiles from Plastic Wastes.

9. Ugoamadi CC, Ihesiulor OK (2011) Optimization of the development of a plastic recycling machine. Nigeria Journal of Technology 30: 67-81.

10. Sharma PD (2008) Plastic waste-reduce, reuse and recycle of plastic wastes are essential to make the Environment Greener and Safer.

11. Kuruppuge UA (1998) Occupational exposure to wood dust. University of Sydney, New South Wales, Australia.

12. Is Wood Biodegradable. 